\title{
Spontaneous Cutaneous Endometriosis of the Umbilicus
}

\author{
Thomas J. Gin ${ }^{a}$ Alexander D. Gin ${ }^{a} \quad$ Douglas Gin ${ }^{b} \quad$ Alan Pham \\ Jennifer Cahill ${ }^{\mathrm{b}}$ \\ ${ }^{a}$ The Royal Melbourne Hospital and Departments of ${ }^{b}$ Dermatology and ${ }^{c}$ Pathology, \\ The Alfred Hospital, Parkville, Vic., Australia
}

\section{Key Words}

Cutaneous endometriosis · Primary endometriosis · Umbilicus

\begin{abstract}
Cutaneous endometriosis that arises de novo, without a prior history of surgery, is a rare phenomenon. The clinical diagnosis of cutaneous endometriosis remains challenging due to the variable clinical appearance and symptoms of the condition, and therefore must be considered in the differential diagnosis of any umbilical lesion. We report a 31-year-old woman who presented with spontaneous cutaneous endometriosis of the umbilicus.
\end{abstract}

(C) 2013 S. Karger AG, Basel

\section{Introduction}

Endometriosis is a common gynaecological condition characterised by the presence of endometrial glands and stroma at extrauterine sites. Ectopic endometrial implants have been reported in nearly every tissue or organ, including the skin. Of the reported cases of cutaneous endometriosis, over $70 \%$ are phenomena secondary to prior surgery and occur at the site of surgical scars [1]. The case of primary cutaneous endometriosis described here remains a rare entity with a reported incidence of $0.5-1 \%$ of all extragenital endometrial ectopia [2]. 


\section{Case Report}

In December 2012, a 31-year-old Caucasian woman presented to the dermatology outpatient clinic with a 1-month history of a persistent, raised lesion in the umbilicus. The patient had noted swelling and one episode of spontaneous frank bleeding from the lesion. There was no associated cyclical pain at the lesion site.

At the time of examination, a single firm $0.5-\mathrm{cm}$ black-coloured papule was seen within the umbilicus at the 3 o'clock position. The lesion was tender to palpation with intact overlying skin. No discharge or underlying umbilical hernia was noted.

The patient had a history of 2 previous melanomas: the first in 2003 on the left abdomen and the second on the right buttock in 2006. Both tumours were excised with clear margins. There was no history of previous abdominal or laparoscopic procedures. She had no previous diagnosis of endometriosis and no catamenial complaints.

Given the anatomical location, a skin punch biopsy of the umbilical lesion was performed. During dermatology review, 1 month later, it was noted that 3 distinct skin-coloured papules had appeared next to the initial lesion (fig. 1).

Histological examination of the punch biopsy revealed a lesion in the superficial dermis comprising a single dilated glandular structure, surrounded by cellular endometrial-type stroma (fig. 2). The gland was lined by a single layer of columnar cells, a few of which had apical cilia. The overlying epidermis was unremarkable. No malignancy was identified. A diagnosis of cutaneous endometriosis was determined.

The patient was referred for surgical excision of the umbilical papules. Subsequent gynaecological evaluation revealed a 3-year history of dysmenorrhoea. A provisional diagnosis of coexistent pelvic endometriosis was made. Abdominal and transvaginal ultrasonography were unremarkable. A diagnostic and therapeutic laparoscopic examination was advised if the patient remained unable to conceive over the following 6 months.

\section{Discussion}

Cutaneous endometriosis accounts for less than $5.5 \%$ of all endometriosis cases [3]. Less than $30 \%$ of cutaneous endometriosis appears in the absence of a prior surgical history and is termed spontaneous (primary) cutaneous endometriosis [1]. Of these cases, the most common site involved is the umbilicus.

Umbilical endometriosis characteristically occurs in women of reproductive age, who present with an umbilical nodule at the site of a prior surgical incision associated with cyclical pain at the lesion site [4]. However, the inconsistent appearance and rarity of this phenomenon means it is often clinically misdiagnosed. Endometriomas under the skin have been described as red, blue, black or flesh-coloured. Lesions may present as single or multiple distinct papules or nodules [5]. Classic associated symptoms include cyclical pain, discharge, bleeding or swelling of the lesion correlated with the menstrual cycle. However, lesions may be entirely asymptomatic [1].

The most widely accepted pathogenesis of secondary endometriosis is the iatrogenic implantation of endometrial cells as a result of surgery, commonly laparoscopic procedures. However, the pathogenesis of primary endometriosis remains uncertain. Theories suggested include the implantation of cells through sanguineous or lymphatic spread, or differentiation from coelomic pluripotent cells in the skin [6].

The heterogeneity in the clinical presentation of cutaneous umbilical endometriosis reflects the breadth of differential diagnoses that may mimic the condition (table 1). 
Gin et al.: Spontaneous Cutaneous Endometriosis of the Umbilicus

Malignancies, in particular melanoma and umbilical metastasis of visceral carcinoma (Sister Mary Joseph nodule), must be considered. Distinctive dermatoscopic [7], ultrasonographic and magnetic resonance imaging findings [8] have been described; however, histopathological examination remains the gold standard. The diagnosis must be confirmed histologically to exclude malignancy.

Definitive treatment is surgical excision. Hormonal therapy in the form of gonadotropinreleasing hormone agonists, oral contraceptives and danazol may be given preoperatively to reduce the size of lesions and ameliorate symptoms consistent with pelvic endometriosis. Subsequent gynaecological evaluation for pelvic endometriosis is recommended for all patients. Around $15 \%$ of patients will have coexistent pelvic endometriosis [9]. In this case, diagnosis of the umbilical skin lesion facilitated the provisional diagnosis and management of underlying symptomatic pelvic endometriosis.

Of note, the patient in this case study has a strong history of melanoma. Recent data have reported a statistically significant association between endometriosis and the occurrence of melanoma [10]. Further studies are required to examine the possible association of cutaneous endometriosis and the development of melanoma.

In summary, spontaneous cutaneous endometriosis remains a rare phenomenon. Nevertheless, it should be considered in the differential diagnosis when examining any umbilical lesion, even in absence of pathognomonic cyclical symptoms. A high index of suspicion will lead to opportune diagnosis and excision. Gynaecologic evaluation after excision is required in all patients to identify underlying pelvic endometriosis.

\section{Disclosure Statement}

The authors declare no conflicts of interest in connection with this work.

\section{References}

1 Fernández-Aceñero MJ, Córdova S: Cutaneous endometriosis: review of 15 cases diagnosed at a single institution. Arch Gynecol Obstet 2011;283:1041-1044.

-2 Latcher JW: Endometriosis of the umbilicus. Am J Obstet Gynecol 1953;66:161-168.

-3 Kyamidis K, Lora V, Kanitakis J: Spontaneous cutaneous umbilical endometriosis: report of a new case with immunohistochemical study and literature review. Dermatol Online J 2011;17:5.

4 Khaled A, Hammami H, Fazaa B, Zermani R, Ben Jilani S, Kamoun MR: Primary umbilical endometriosis: a rare variant of extragenital endometriosis. Pathologica 2008;100:473-475.

5 Weller CV: Endometriosis of the umbilicus. Am J Pathol 1935;11:281-286.1.

-6 Frischknecht F, Raio L, Fleischmann A, Dreher E, Lüscher KP, Mueller MD: Umbilical endometriosis. Surg Endosc 2004;18:347.

7 De Giorgi V, Massi D, Mannone F, Stante M, Carli P: Cutaneous endometriosis: non-invasive analysis by epiluminescence microscopy. Clin Exp Dermatol 2003;28:315-317.

-8 Hartigan CM, Holloway BJ: Case report: MR imaging features of endometriosis at the umbilicus. Br J Radiol 2005;78:755-757.

-9 Dadhwal V, Gupta B, Dasgupta C, Shende U, Deka D: Primary umbilical endometriosis: a rare entity. Arch Gynecol Obstet 2011;283(suppl 1):119-120.

10 Kvaskoff M, Mesrine S, Fournier A, Boutron-Ruault M, Clavel-Chapelon F: Personal history of endometriosis and risk of cutaneous melanoma in a large prospective cohort of French women. Arch Intern Med 2007;167:2061-2065. 
Gin et al.: Spontaneous Cutaneous Endometriosis of the Umbilicus

Table 1. The potential differential diagnoses to be considered when evaluating an umbilical nodule

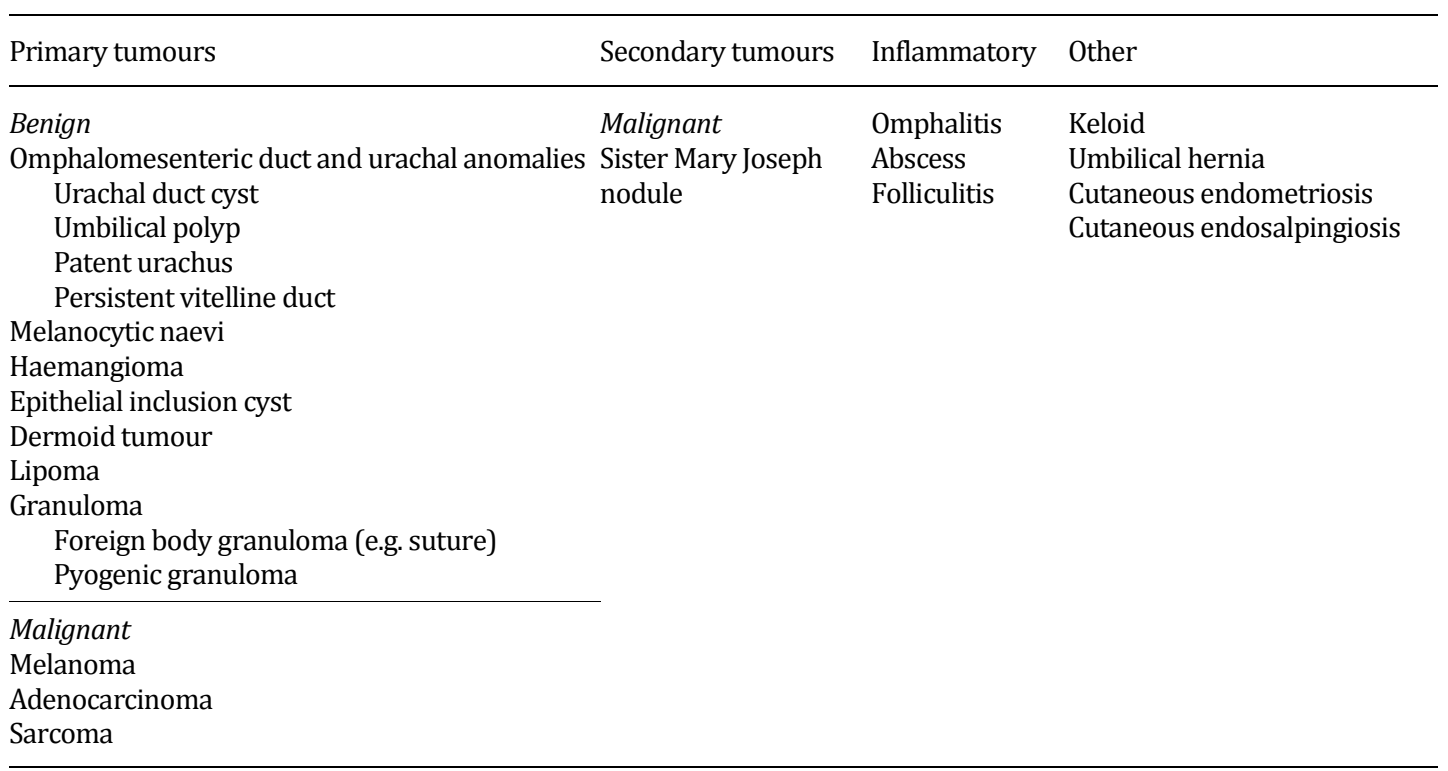

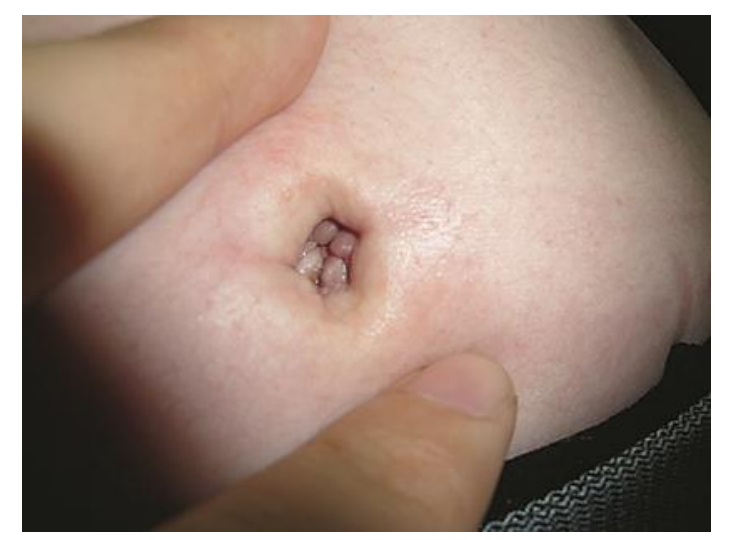

Fig. 1. Second presentation, 1 month after umbilical papule punch biopsy. Three distinct skin-coloured papules had appeared next to the initial lesion. 


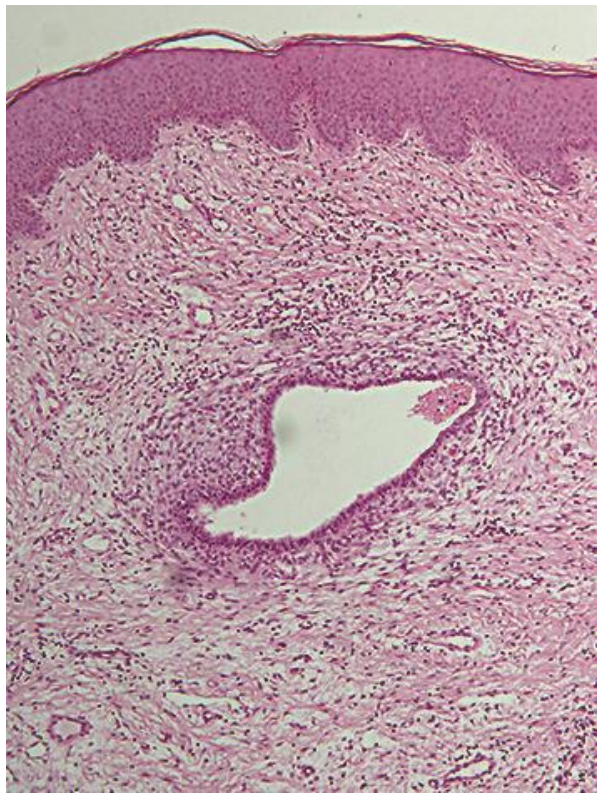

Fig. 2. Histopathological imaging of punch biopsy demonstrating a lesion in the superficial dermis comprising a single dilated glandular structure, surrounded by cellular endometrial-type stroma (HE, $\times 100$ ). 